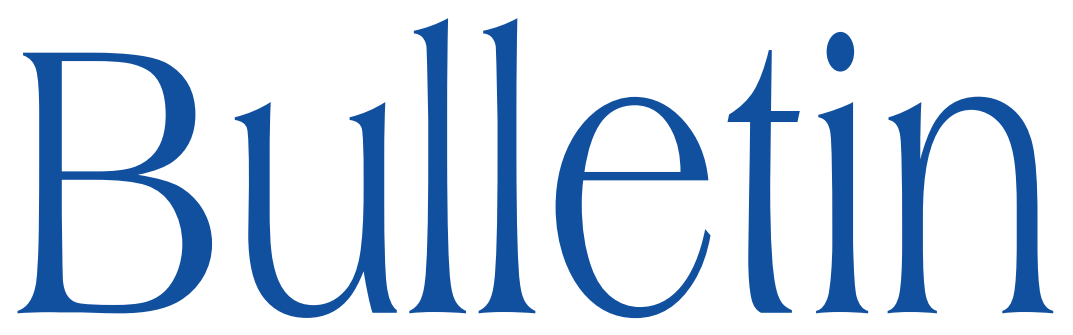

de la SOCIÉTÉ MATHÉMATIQUE DE FRANCE

\title{
BATALIN-VILKOVISKY ALGEBRA STRUCTURES ON HOCHSCHILD COHOMOLOGY
}

\author{
Luc Menichi
}

\section{Tome 137}

Fascicule 2

2000 


\title{
BATALIN-VILKOVISKY ALGEBRA STRUCTURES ON HOCHSCHILD COHOMOLOGY
}

\author{
BY LUC MENICHI
}

\begin{abstract}
Let $M$ be any compact simply-connected oriented $d$-dimensional smooth manifold and let $\mathbb{F}$ be any field. We show that the Gerstenhaber algebra structure on the Hochschild cohomology on the singular cochains of $M$, $H H^{*}\left(S^{*}(M), S^{*}(M)\right)$, extends to a Batalin-Vilkovisky algebra. Such BatalinVilkovisky algebra was conjectured to exist and is expected to be isomorphic to the Batalin-Vilkovisky algebra on the free loop space homology on $M, H_{*+d}(L M)$ introduced by Chas and Sullivan. We also show that the negative cyclic cohomology $H C_{-}^{*}\left(S^{*}(M)\right)$ has a Lie bracket. Such Lie bracket is expected to coincide with the Chas-Sullivan string bracket on the equivariant homology $H_{*}^{S^{1}}(L M)$.

RÉSUMÉ (Structures d'algèbres de Batalin-Vilkovisky sur la cohomologie de Hochschild)

Soit $M$ une variété lisse orientée compact simplement connexe de dimension $d$. Soit $\mathbb{F}$ un corps commutatif quelconque. Nous montrons que la structure d'algèbre de Gerstenhaber sur la cohomologie de Hochschild des cochaînes singulières de $M$, $H H^{*}\left(S^{*}(M), S^{*}(M)\right)$, s'étend en une algèbre de Batalin-Vilkovisky. L'existence d'une telle algèbre de Batalin-Vilkovisky était conjecturée. Il est prévu qu'une telle algèbre soit isomorphe à l'algèbre de Batalin-Vilkovisky sur l'homologie des lacets libres sur $M$, $H_{*+d}(L M)$, introduite par Chas and Sullivan. Nous montrons aussi que la cohomologie cyclique négative $H C_{-}^{*}\left(S^{*}(M)\right)$ possède un crochet de Lie. Ce crochet de Lie devrait coincider avec le crochet des cordes de Chas et Sullivan sur l'homologie équivariante $H_{*}^{S^{1}}(L M)$.
\end{abstract}

Texte reçu le 12 mai 2008, révisé le 28 octobre 2008, accepté le 13 novembre 2008

Luc Menichi, UMR 6093 associée au CNRS, Université d'Angers, Faculté des Sciences, 2 Boulevard Lavoisier, 49045 Angers, France - E-mail : luc.menichi@univ-angers.fr 2000 Mathematics Subject Classification. - 16E40, 16E45, 55P35, 57P10.

Key words and phrases. - String topology, Batalin-Vilkovisky algebra, Gerstenhaber algebra, Hochschild cohomology, free loop space. 


\section{Introduction}

Except where specified, we work over an arbitrary field $\mathbb{F}$. Let $M$ be a compact oriented $d$-dimensional smooth manifold. Denote by $L M:=\operatorname{map}\left(S^{1}, M\right)$ the free loop space on $M$. Chas and Sullivan [1] have shown that the shifted free loop homology $H_{*+d}(L M)$ has a structure of Batalin-Vilkovisky algebra (Definition 8). In particular, they showed that $H_{*+d}(L M)$ is a Gerstenhaber algebra (Definition 7). On the other hand, let $A$ be a differential graded (unital associative) algebra. The Hochschild cohomology of $A$ with coefficients in $A$, $H H^{*}(A, A)$, is a Gerstenhaber algebra. These two Gerstenhaber algebras are expected to be related:

Conjecture 1 (due to [1, "dictionary" p. 5] or [4]?). - If $M$ is simply connected then there is an isomorphism of Gerstenhaber algebras $H_{*+d}(L M) \cong$ $H H^{*}\left(S^{*}(M), S^{*}(M)\right)$ between the free loop space homology and the Hochschild cohomology of the algebra of singular cochains on $M$.

Félix, Thomas and Vigué-Poirrier [13, Section 7] proved that there is a linear isomorphism of lower degree $d$ (See notation 6 for our degree conventions).

$$
\mathbb{D}: H H^{-p-d}\left(S^{*}(M), S^{*}(M)^{\vee}\right) \stackrel{\cong}{\rightarrow} H H^{-p}\left(S^{*}(M), S^{*}(M)\right) \text {. }
$$

We prove

Theorem 3 (Theorem 22). - The Connes coboundary map on $H H^{*}\left(S^{*}(M), S^{*}(M)^{\vee}\right)$ defines via the isomorphism (2) a structure of BatalinVilkovisky algebra extending the Gerstenhaber algebra $H H^{*}\left(S^{*}(M), S^{*}(M)\right)$.

Assume that $M$ is simply-connected. Jones [19] proved that there is an isomorphism

$$
J: H_{p+d}(L M) \stackrel{\cong}{\rightarrow} H H^{-p-d}\left(S^{*}(M), S^{*}(M)^{\vee}\right)
$$

such that the $\Delta$ operator of the Batalin-Vilkovisky algebra $H_{*+d}(L M)$ and Connes coboundary map $B^{\vee}$ on $H H^{*-d}\left(S^{*}(M), S^{*}(M)^{\vee}\right)$ satisfies $J \circ \Delta=$ $B^{\vee} \circ J$. Of course, we conjecture:

Conjecture 4. - The isomorphism

$$
\mathbb{D} \circ J: H_{p+d}(L M) \stackrel{\cong}{\rightarrow} H H^{-p}\left(S^{*}(M), S^{*}(M)\right)
$$

is a morphism of graded algebras. 
Notice that Conjecture 4 implies that the composite $\mathbb{D} \circ J$ is an isomorphism of Batalin-Vilkovisky algebras between the Chas-Sullivan Batalin-Vilkovisky algebra and the Batalin-Vilkovisky algebra defined by Theorem 22. Therefore Conjecture 4 implies Conjecture 1.

Cohen and Jones [4, Theorem 3] first mentioned an isomorphism of algebras

$$
H_{p+d}(L M) \stackrel{\cong}{\rightrightarrows} H H^{-p}\left(S^{*}(M), S^{*}(M)\right) \text {. }
$$

Over the reals or over the rationals, two proofs of such an isomorphism of graded algebras have been given by Merkulov [25] and Félix, Thomas, ViguéPoirrier [14].

Theorem 22 comes from a general result (Propositions 11 and 12) which shows that the Hochschild cohomology $H^{*}(A, A)$ of a differential graded algebra $A$ which is a "symmetric algebra in the derived category", is a BatalinVilkovisky algebra. As second application of this general result, we recover the following theorem due to Thomas Tradler.

Theorem 5 ([26, Example 2.15 and Theorem 3.1] (Corollary 19))

Let $A$ be a symmetric algebra. Then $H H^{*}(A, A)$ is a Batalin-Vilkovisky algebra.

This theorem has been reproved and extended by many people [5, 8, 18, 20, 21, 22, 23, 28] (in chronological order). The last proof, the proof of Eu et Schedler [8] looks similar to ours.

Thomas Tradler gave a somehow complicated proof of the previous theorem (Corollary 19). Indeed, his goal was to prove our main theorem (Theorem 22). In [29] or in [27], Tradler and Zeinalian proved Theorem 22 but only over a field of characteristic 0 [29, "rational simplicial chain" in the abstract] or [27, Beginning of 3.1]. Costello's result [5, Section 2.1] is also over a field of characteristic 0 .

Over $\mathbb{Q}$, we explain in Corollary 20 how to put a Batalin-Vilkovisky algebra structure on $H H^{*}\left(S^{*}(M ; \mathbb{Q}), S^{*}(M ; \mathbb{Q})\right)$ from a slight generalisation of Corollary 19 (Theorem 18). In fact both Félix, Thomas [12] and Chen [3, Theorem $5.4]$ proved that the Chas-Sullivan Batalin-Vilkovisky algebra $H_{*+d}(L M ; \mathbb{Q})$ is isomorphic to the Batalin-Vilkovisky algebra given by Corollary 20 .

Remark that, over $\mathbb{Q}$, when the manifold $M$ is formal, a consequence of Félix and Thomas work [12], is that $H_{*+d}(L M)$ is always isomorphic to the BatalinVilkovisky algebra $H H^{*}\left(H^{*}(M) ; H^{*}(M)\right)$ given by Corollary 19 applied to the symmetric algebra $H^{*}(M)$. Over $\mathbb{F}_{2}$, in [24], we showed that this is not the case. The present paper seems to explain why:

The Batalin-Vilkovisky algebra on $H H^{*}\left(S^{*}(M), S^{*}(M)\right)$ given by Theorem 22 depends of course on the algebra $S^{*}(M)$ but also on a fundamental 
class $[m] \in H H^{-d}\left(S^{*}(M), S^{*}(M)^{\vee}\right)$ which seems hard to compute. This fundamental class $[m]$ involves chain homotopies for the commutativity of the algebra $S^{*}(M)$.

The Batalin-Vilkovisky algebra on $H H^{*}\left(S^{*}(M ; \mathbb{Q}), S^{*}(M ; \mathbb{Q})\right)$ given by Corollary 20, depends of

- a commutative algebra, Sullivan's cochain algebra of polynomial differential forms $A_{P L}(M)$ [10],

- and of the fundamental class $[M] \in H_{d}\left(A_{P L}(M)^{\vee}\right)$.

Acknowledgment. - We wish to thank Jean-Claude Thomas for a discussion concerning Ginzburg's preprint [17]. We would like also to thank Yves Félix for explaining us the wonderful isomorphism (2).

\section{Hochschild homology and cohomology}

We use the graded differential algebra of [10, Chapter 3]. In particular, an element of lower degree $i \in \mathbb{Z}$ is by the classical convention [10, p. 41-2] of upper degree $-i$. Differentials are of lower degree -1 . All the algebras considered in this paper, are unital and associative. Let $A$ be a differential graded algebra. Denote by $s A$ the suspension of $A,(s A)_{i}=A_{i-1}$. Let $d_{0}$ be the differential on the tensor product of complexes $A \otimes T(s A) \otimes A$. We denote the tensor product of the elements $a \in A, s a_{1} \in s A, \ldots, s a_{k} \in s A$ and $b \in A$ by $a\left[a_{1}|\cdots| a_{k}\right] b$. Let $d_{1}$ be the differential on the graded vector space $A \otimes T(s A) \otimes A$ defined by:

$$
\begin{aligned}
d_{1} a\left[a_{1}|\cdots| a_{k}\right] b= & (-1)^{|a|} a a_{1}\left[a_{2}|\cdots| a_{k}\right] b \\
& +\sum_{i=1}^{k-1}(-1)^{\varepsilon_{i}} a\left[a_{1}|\cdots| a_{i} a_{i+1}|\cdots| a_{k}\right] b \\
& -(-1)^{\varepsilon_{k-1}} a\left[a_{1}|\cdots| a_{k-1}\right] a_{k} b ;
\end{aligned}
$$

Here $\varepsilon_{i}=|a|+\left|a_{1}\right|+\cdots+\left|a_{i}\right|+i$. The bar resolution of $A$, denoted $B(A ; A ; A)$, is the differential graded $(A, A)$-bimodule $\left(A \otimes T(s A) \otimes A, d_{0}+d_{1}\right)$.

Denote by $A^{\text {op }}$ the opposite algebra of $A$. Recall that any $(A, A)$-bimodule can be considered as a left (or right) $A \otimes A^{\mathrm{op}}$-module. The Hochschild chain complex is the complex $A \otimes_{A \otimes A^{\text {op }}} B(A ; A ; A)$ denoted $\mathcal{C}_{*}(A, A)$. Explicitly $\mathcal{C}_{*}(A, A)$ is the complex $\left(A \otimes T(s A), d_{0}+d_{1}\right)$ with $d_{0}$ obtained by tensorization 
and $[6,(10)$ p. 78$]$

$$
\begin{aligned}
d_{1} a\left[a_{1}|\cdots| a_{k}\right]= & (-1)^{|a|} a a_{1}\left[a_{2}|\cdots| a_{k}\right] \\
& +\sum_{i=1}^{k-1}(-1)^{\varepsilon_{i}} a\left[a_{1}|\cdots| a_{i} a_{i+1}|\cdots| a_{k}\right] \\
& -(-1)^{\left|s a_{k}\right| \varepsilon_{k-1}} a_{k} a\left[a_{1}|\cdots| a_{k-1}\right] .
\end{aligned}
$$

The Hochschild homology is the homology $H$ of the Hochschild chain complex:

$$
H H_{*}(A, A):=H\left(\mathcal{C}_{*}(A, A)\right) .
$$

Let $M$ be a differential graded $(A, A)$-bimodule. The Hochschild cochain complex of $A$ with coefficients in $M$ is the complex $\operatorname{Hom}_{A \otimes A^{\text {op }}}(B(A ; A ; A), M)$ denoted $\mathcal{C}^{*}(A, M)$. Explicitly $\mathcal{C}^{*}(A, M)$ is the complex

$$
\left(\operatorname{Hom}(T(s A), M), D_{0}+D_{1}\right) \text {. }
$$

Here for $f \in \operatorname{Hom}(T(s A), M), D_{0}(f)([])=d_{M}(f([])), D_{1}(f)([])=0$, and for $k \geq 1$, we have:

$$
D_{0}(f)\left(\left[a_{1}\left|a_{2}\right| \ldots \mid a_{k}\right]\right)=d_{M}\left(f\left(\left[a_{1}\left|a_{2}\right| \ldots \mid a_{k}\right]\right)\right)-\sum_{i=1}^{k}(-1)^{\bar{\epsilon}_{i}} f\left(\left[a_{1}|\ldots| d_{A} a_{i}|\ldots| a_{k}\right]\right)
$$

and

$$
\begin{aligned}
D_{1}(f)\left(\left[a_{1}\left|a_{2}\right| \ldots \mid a_{k}\right]\right)= & -(-1)^{\left|s a_{1}\right||f|} a_{1} f\left(\left[a_{2}|\ldots| a_{k}\right]\right) \\
& -\sum_{i=2}^{k}(-1)^{\bar{\epsilon}_{i}} f\left(\left[a_{1}|\ldots| a_{i-1} a_{i}|\ldots| a_{k}\right]\right) \\
& +(-1)^{\bar{\epsilon}_{k}} f\left(\left[a_{1}\left|a_{2}\right| \ldots \mid a_{k-1}\right]\right) a_{k},
\end{aligned}
$$

where $\bar{\epsilon}_{i}=|f|+\left|s a_{1}\right|+\left|s a_{2}\right|+\ldots+\left|s a_{i-1}\right|$.

The Hochschild cohomology of $A$ with coefficients in $M$ is

$$
H H^{*}(A, M)=H\left(\mathcal{C}^{*}(A, M)\right) \text {. }
$$

Since we work over an arbitrary field $\mathbb{F}$, the bar resolution $B(A ; A ; A) \stackrel{\simeq}{\rightarrow} A$ is a semi-free resolution of $A$ as an $(A, A)$-bimodule (same proof as in the normalized case [10, Proposition 19.2(ii)]). Therefore the Hochschild homology of $A$ is the differential torsion product

$$
H H_{*}(A, A)=\operatorname{Tor}^{A \otimes A^{\mathrm{op}}}(A, A)
$$

and the Hochschild cohomology is

$$
H H^{*}(A, M)=\operatorname{Ext}_{A \otimes A^{\text {op }}}(A, M)
$$

where the latter denotes the differential "Ext" in the sense of J.C. Moore (cf. [9, Appendix]). 
Notation 6. - Let $n \in \mathbb{Z}$ be an integer. We denote by $H H^{n}(A, M)$ the set $\left[H H^{*}(A, M)\right]^{n}$ of homogeneous elements of upper degree $n$ in the graded vector space $H H^{*}(A, M)$. We denote also by $H H_{n}(A, A)$ the set $\left[H H_{*}(A, A)\right]_{n}$ of homogeneous elements of lower degree $n$ in the graded vector space $H H_{*}(A, A)$. Therefore, $H H^{*}(A, M)$ is the upper graded vector space $\left\{H H^{n}(A, M)\right\}_{n \in \mathbb{Z}}$ and $H H_{*}(A, A)$ is the lower graded vector space $\left\{H H_{n}(A, A)\right\}_{n \in \mathbb{Z}}$. If $A$ and $M$ are concentrated in degree 0 , theses notations coincide with the usual ones.

Gerstenhaber proved that the Hochschild cohomology of $A$ with coefficients in $A, H H^{*}(A, A)$, is a Gerstenhaber algebra [16].

Definition 7. - A Gerstenhaber algebra is a commutative graded algebra $A$ equipped with a linear map $\{-,-\}: A_{i} \otimes A_{j} \rightarrow A_{i+j+1}$ of degree 1 such that:

a) the bracket $\{-,-\}$ gives $A$ a structure of graded Lie algebra of degree 1 . This means that for each $a, b$ and $c \in A$

$$
\begin{aligned}
& \{a, b\}=-(-1)^{(|a|+1)(|b|+1)}\{b, a\} \text { and } \\
& \{a,\{b, c\}\}=\{\{a, b\}, c\}+(-1)^{(|a|+1)(|b|+1)}\{b,\{a, c\}\} .
\end{aligned}
$$

b) the product and the Lie bracket satisfy the following relation called the Poisson relation:

$$
\{a, b c\}=\{a, b\} c+(-1)^{(|a|+1)|b|} b\{a, c\} .
$$

In this paper, we show that for some algebras $A$, the Gerstenhaber algebra structure of $H H^{*}(A, A)$ extends to a Batalin-Vilkovisky algebra.

Definition 8. - A Batalin-Vilkovisky algebra is a Gerstenhaber algebra $A$ equipped with a degree 1 linear map $\Delta: A_{i} \rightarrow A_{i+1}$ such that $\Delta \circ \Delta=0$ and such that the bracket is given by

$$
\{a, b\}=(-1)^{|a|}\left(\Delta(a \cup b)-(\Delta a) \cup b-(-1)^{|a|} a \cup(\Delta b)\right)
$$

for $a$ and $b \in A$.

\section{The isomorphism between $H H^{*}(A, A)$ and $H H^{*}\left(A, A^{\vee}\right)$}

Proposition 11 gives an isomorphism between the Hochschild cohomology of $A$ with coefficients in $A, H H^{*}(A, A)$ and the Hochschild cohomology of $A$ with coefficients in the dual $A^{\vee}, H H^{*}\left(A, A^{\vee}\right)$. To obtain an isomorphism $H H^{*}(A, A) \cong H H^{*}\left(A, A^{\vee}\right)$, one could ask for an isomorphism or a quasiisomorphism of $A$-bimodules $\Theta: A \stackrel{\simeq}{\rightrightarrows} A^{\vee}$. But this condition is too strong. Instead, we are going to ask just for a derived isomorphism of $(A, A)$-bimodules $m$ from $A$ to $A^{\vee}$. 
Let $m$ be any derived morphism of $(A, A)$-bimodules from $A$ to $A^{\vee}$. The hypothesis of Proposition 11 is a sufficient (and in fact necessary) condition for $m$ to be a quasi-isomorphism (=isomorphism in the derived category).

This method for obtaining an isomorphism $H H^{*}(A, A) \cong H H^{*}\left(A, A^{\vee}\right)$ is a generalisation of the method used by Félix, Thomas and Vigué-Poirrier in the case $A=S^{*}(M)$ to obtain the isomorphism (2).

Then we show that this isomorphism looks like a Poincaré duality isomorphism: this isomorphism is given by the action of the algebra $H H^{*}(A, A)$ on a fundamental class $[m] \in H H^{*}\left(A, A^{\vee}\right)$.

Let us first recall the definition of the action of $H H^{*}(A, A)$ on $H H^{*}\left(A, A^{\vee}\right)$. Let $A$ be a (differential graded) algebra. Let $M$ and $N$ be two $A$-bimodules. Let $f \in \mathcal{C}^{*}(A, M)$ and $g \in \mathcal{C}^{*}(A, N)$. We denote by $\otimes_{A}(f, g) \in \mathcal{C}^{*}\left(A, M \otimes_{A} N\right)$ the linear map defined by

$$
\otimes_{A}(f, g)\left(\left[a_{1}|\ldots| a_{n}\right]\right)=\sum_{p=0}^{n} \pm f\left(\left[a_{1}|\ldots| a_{p}\right]\right) \otimes_{A} g\left(\left[a_{p+1}|\ldots| a_{n}\right]\right)
$$

Here \pm is the Koszul sign $(-1)^{|g|\left(\left|a_{1}\right|+\ldots\left|a_{p}\right|+p\right)}$. This define a natural morphism of complexes

$$
\otimes_{A}: \mathcal{C}^{*}(A, M) \otimes \mathcal{C}^{*}(A, N) \rightarrow \mathcal{C}^{*}\left(A, M \otimes_{A} N\right)
$$

Therefore, in homology, we have a natural morphism

$$
H_{*}\left(\otimes_{A}\right): H H^{*}(A, M) \otimes H H^{*}(A, N) \rightarrow H H^{*}\left(A, M \otimes_{A} N\right)
$$

If we let take $A=M$, and use the isomorphism of $A$-bimodules

$$
A \otimes_{A} N \stackrel{\cong}{\rightrightarrows} N, a \otimes_{A} n \mapsto a . n,
$$

the composite

$$
\mathcal{C}^{*}(A, A) \otimes \mathcal{C}^{*}(A, N) \stackrel{\otimes_{A}}{\rightarrow} \mathcal{C}^{*}\left(A, A \otimes_{A} N\right) \cong \mathcal{C}^{*}(A, N)
$$

is a left action of $\mathcal{C}^{*}(A, A)$ on $\mathcal{C}^{*}(A, N)$. In the particular case, $A=M=N$, this composite is the usual cup product on $\mathcal{C}^{*}(A, A)$ denoted $\cup$.

Denote by $A^{\vee}$ the dual of $A$. Let $\eta: \mathbb{F} \rightarrow A$ be the unit of the algebra. Then we have a natural map

$$
H H^{*}\left(\eta, A^{\vee}\right): H H^{*}\left(A, A^{\vee}\right) \rightarrow H H^{*}\left(\mathbb{F}, A^{\vee}\right) \cong H\left(A^{\vee}\right) .
$$

Proposition 11. - Let $[m] \in H H^{-d}\left(A, A^{\vee}\right)$ be an element of lower degree $d$ such that the morphism of left $H(A)$-modules

$$
H(A) \stackrel{\cong}{\rightrightarrows} H\left(A^{\vee}\right), a \mapsto a \cdot H H^{-d}\left(\eta, A^{\vee}\right)([m])
$$


is an isomorphism. Then the action of $H H^{*}(A, A)$ on $[m] \in H H^{-d}\left(A, A^{\vee}\right)$ gives the isomorphism of lower degree $d$ of $\mathrm{HH}^{*}(A, A)$-modules

$$
H H^{p}(A, A) \stackrel{\cong}{\rightrightarrows} H H^{p-d}\left(A, A^{\vee}\right), a \mapsto a \cdot[m] .
$$

Proof. - Let $\varepsilon_{A}: P \stackrel{\simeq}{\rightarrow} A$ be a resolution of $A$ as left $A \otimes A^{\text {op }}$-semifree module. Let $s_{A}: A \stackrel{\simeq}{\leftrightarrows} P$ be a morphism of left $A$-modules which is a section of $\varepsilon_{A}$. The morphism $H H^{*}\left(\eta, A^{\vee}\right): H H^{*}\left(A, A^{\vee}\right) \rightarrow H H^{*}\left(\mathbb{F}, A^{\vee}\right)$ is equal to the following composite of

$$
H H^{*}\left(A, A^{\vee}\right):=\operatorname{Ext}_{A \otimes A^{\mathrm{op}}}\left(A, A^{\vee}\right) \stackrel{\operatorname{Ext}_{i_{1}}\left(A, A^{\vee}\right)}{\rightarrow} \operatorname{Ext}_{A}\left(A, A^{\vee}\right)
$$

and

$$
\operatorname{Ext}_{A}\left(A, A^{\vee}\right) \stackrel{\operatorname{Ext}_{\eta}\left(\eta, A^{\vee}\right)}{\cong} \operatorname{Ext}_{\mathbb{F}}\left(\mathbb{F}, A^{\vee}\right)=: H H^{*}\left(\mathbb{F}, A^{\vee}\right)
$$

where $i_{1}: A \hookrightarrow A \otimes A^{\text {op }}$ is the inclusion of the first factor.

Therefore, $H H^{*}\left(\eta, A^{\vee}\right)$ is the map induced in homology by the composite

$$
\operatorname{Hom}_{A \otimes A^{\mathrm{op}}}\left(P, A^{\vee}\right) \stackrel{\operatorname{Hom}\left(s_{A}, A^{\vee}\right)}{\rightarrow} \operatorname{Hom}_{A}\left(A, A^{\vee}\right) \underset{\cong}{\stackrel{e v\left(1_{A}\right)}{\longrightarrow}} A^{\vee} .
$$

where $e v\left(1_{A}\right)$ is the evaluation at the unit $1_{A} \in A$. This composite maps the cycle $m \in \operatorname{Hom}_{A \otimes A^{\text {op }}}\left(P, A^{\vee}\right)$ to $m \circ s_{A}$ and then to $\left(m \circ s_{A}\right)\left(1_{A}\right)$. Since $m \circ s_{A}: A \rightarrow A^{\vee}$ maps $a \in A$ to $a \cdot\left(\left(m \circ s_{A}\right)(1)\right)$, by hypothesis, $m \circ s_{A}$ is a quasi-isomorphism. Since $s_{A}$ is a quasi-isomorphism, $m: P \stackrel{\simeq}{\rightarrow} A^{\vee}$ is also a quasi-isomorphism.

By applying the functor $\operatorname{Hom}_{A \otimes A^{\mathrm{op}}}(P,-)$ to the two quasi-isomorphisms of $A$-bimodules

$$
A \stackrel{\varepsilon_{A}}{\stackrel{\leftarrow}{\simeq}} P \underset{\simeq}{\stackrel{m}{\longrightarrow}} A^{\vee}
$$

we obtain the quasi-isomorphism of complexes

$$
\operatorname{Hom}_{A \otimes A^{\mathrm{op}}}(P, A) \underset{\simeq}{\stackrel{\varepsilon_{A}}{\simeq}} \operatorname{Hom}_{A \otimes A^{\mathrm{op}}}(P, P) \stackrel{\operatorname{Hom}_{A \otimes A^{\mathrm{op}}}(P, m)}{\underset{\simeq}{\longrightarrow}} \operatorname{Hom}_{A \otimes A^{\mathrm{op}}}\left(P, A^{\vee}\right) .
$$

By applying homology, we get the desired isomorphism, since the action of $H H^{*}(A, A)$ on $H H^{*}\left(A, A^{\vee}\right)$ is induced by the composition map

$$
\begin{gathered}
\operatorname{Hom}_{A \otimes A^{\mathrm{op}}}\left(P, A^{\vee}\right) \otimes \operatorname{Hom}_{A \otimes A^{\mathrm{op}}}(P, P) \rightarrow \operatorname{Hom}_{A \otimes A^{\mathrm{op}}}\left(P, A^{\vee}\right) \\
m \otimes f \mapsto m \circ f=\operatorname{Hom}_{A \otimes A^{\mathrm{op}}}(P, m)(f)
\end{gathered}
$$

Alternatively, the two isomorphisms

$$
H H^{*}(A, A) \stackrel{H *\left(A, \varepsilon_{A}\right)^{-1}}{\cong} H H^{*}(A, P) \stackrel{H H^{*}(A, m)}{\cong} H H^{*}\left(A, A^{\vee}\right)
$$

maps $\left[\varepsilon_{A}\right]$ (which is the unit of $H H^{*}(A, A)$ ) to $\left[i d_{P}: P \rightarrow P\right]$ and then to $[m]$. They are morphisms of $H H^{*}(A, A)$-modules since

$$
H_{*}\left(\otimes_{A}\right): H H^{*}(A, A) \otimes H H^{*}(A, N) \rightarrow H H^{*}\left(A, A \otimes_{A} N\right)
$$


is natural with respect to $N$.

\section{Batalin-Vilkovisky algebra structures on Hochschild cohomology}

In this section, we explain when an isomorphism $H H^{*}(A, A) \cong H H^{*}\left(A, A^{\vee}\right)$ gives a Batalin-Vilkovisky algebra structure on the Gerstenhaber algebra $H H^{*}(A, A)$. Our proof relies on the proof of a similar result due to Ginzburg [17, Theorem 3.4.3 (ii)]. Ginzburg basically explains when an isomorphism $H H^{*}(A, A) \cong H H_{*}(A, A)$ gives a Batalin-Vilkovisky algebra structure on $H H^{*}(A, A)$.

Denote by $B$ Connes boundary in the Hochschild complex $\mathcal{C}_{*}(A, A)$ and by $B^{\vee}$ its dual in $\mathcal{C}^{*}\left(A, A^{\vee}\right) \cong \mathcal{C}_{*}(A, A)^{\vee}$. We prove:

Proposition 12. - Let $[m] \in H H^{-d}\left(A, A^{\vee}\right)$ such that the morphism of $H H^{*}(A, A)$-modules

$$
H H^{p}(A, A) \stackrel{\cong}{\rightarrow} H H^{p-d}\left(A, A^{\vee}\right), a \mapsto a \cdot[m]
$$

is an isomorphism. If $H_{*}\left(B^{\vee}\right)([m])=0$ then the Gerstenhaber algebra $H H^{*}(A, A)$ equipped with $H_{*}\left(B^{\vee}\right)$ is a Batalin-Vilkovisky algebra.

As we will see Proposition 12 is almost the dual of the following Proposition due to Victor Ginzburg. Recall first that the Hochschild cohomology of a (differential graded) algebra, acts on its Hochschild homology [6, equation (18) and Proposition 2.6 p. 82]

$$
\begin{gathered}
H H^{p}(A, A) \otimes H H_{d}(A, A) \rightarrow H H_{p-d}(A, A) \\
\eta \otimes c \mapsto i_{\eta}(c)=\eta . c
\end{gathered}
$$

In non-commutative geometry, the action of $\eta \in H H^{*}(A, A)$ on $c \in H H_{*}(A, A)$ is denoted by $i_{\eta}(c)$.

Proposition 13 ([17, Theorem 3.4 .3 (ii)]). - Let $c \in H H_{d}(A, A)$ such that the morphism of $H^{*}(A, A)$-modules

$$
H H^{p}(A, A) \stackrel{\cong}{\rightrightarrows} H H_{d-p}(A, A), \eta \mapsto \eta \cdot c
$$

is an isomorphism. If $H_{*}(B)(c)=0$ then the Gerstenhaber algebra $H H^{*}(A, A)$ equipped with $H_{*}(B)$ is a Batalin-Vilkovisky algebra.

REMARK 14. - The condition $H_{*}(B)(c)=0$ does not appear in [17, Theorem 3.4 .3 (ii)] since according to Ginzburg, this condition is automatically satisfied for a Calabi-Yau algebra of dimension $d$. In both Propositions 12 and 13, if the 
condition $H_{*}\left(B^{\vee}\right)([m])=0$ or $H_{*}(B)(c)=0$ is not satisfied, $\Delta(1)$ can be non zero and the relation (9) is replaced by the more general relation

$$
\begin{aligned}
\{\xi, \eta\}=(-1)^{|\xi|}[\Delta(\xi \cup \eta)- & \\
& \left.(-1)^{|\xi|} \xi \cup(\Delta \eta)-(\Delta \xi) \cup \eta+(-1)^{|\xi|+|\eta|} \xi \cup \eta \cup(\Delta 1)\right] .
\end{aligned}
$$

Proof of Proposition 13. - By definition the $\Delta$ operator on $H H^{*}(A, A)$ is given by $(\Delta a) . c:=B(a . c)$ for any $a \in H H^{*}(A, A)$. Therefore the proposition follows from the following Lemma due to Victor Ginzburg.

Lemma 15 ([17, formula (9.3.2)]). — Let $A$ be a differential graded algebra. For any $\eta, \xi \in H H^{*}(A, A)$ and $c \in H H_{*}(A, A)$,

$$
\begin{aligned}
\{\xi, \eta\} . c=(-1)^{|\xi|} B[(\xi \cup \eta) \cdot c]-\xi \cdot B(\eta \cdot c) & \\
& +(-1)^{(|\eta|+1)(|\xi|+1)} \eta \cdot B(\xi . c)+(-1)^{|\eta|}(\xi \cup \eta) \cdot B(c) .
\end{aligned}
$$

Proof. — Let us recall the proof of Victor Ginzburg. Denote by

$$
\begin{gathered}
H H^{p}(A, A) \otimes H H_{j}(A, A) \rightarrow H H_{j-p+1}(A, A) \\
(\eta, a) \mapsto L_{\eta}(a)
\end{gathered}
$$

the action of the suspended graded Lie algebra $H H^{*}(A, A)$ on $H H_{*}(A, A)$. Gelfand, Daletski and Tsygan [15] proved that the Gerstenhaber algebra $H H^{*}(A, A)$ and Connes boundary map $B$ on $H H_{*}(A, A)$ form a calculus [6, p. 93]. Therefore, we have the following equalities

$$
\begin{aligned}
& i_{\{\xi, \eta\}}=\left\{L_{\xi}, i_{\eta}\right\}=L_{\xi} \circ i_{\eta}-(-1)^{(|\xi|+1)|\eta|} i_{\eta} \circ L_{\xi} \\
= & (-1)^{|\xi|}\left\{B, i_{\xi}\right\} \circ i_{\eta}-(-1)^{(|\xi|+1)|\eta|} i_{\eta} \circ(-1)^{|\xi|}\left\{B, i_{\xi}\right\} \\
= & (-1)^{|\xi|} B \circ i_{\xi} \circ i_{\eta}-i_{\xi} \circ B \circ i_{\eta}+(-1)^{(|\eta|+1)(|\xi|+1)} i_{\eta} \circ B \circ i_{\xi}+(-1)^{|\eta|(|\xi|+1)} i_{\eta} \circ i_{\xi} \circ B \\
= & (-1)^{|\xi|} B \circ i_{\xi \cup \eta}-i_{\xi} \circ B \circ i_{\eta}+(-1)^{(|\eta|+1)(|\xi|+1)} i_{\eta} \circ B \circ i_{\xi}+(-1)^{|\eta|} i_{\xi \cup \eta} \circ B .
\end{aligned}
$$

By applying this equality of operators to $c$, we obtain the Lemma.

We now prove the following Lemma which is the dual of Lemma 15.

Lemma 16. - Let $A$ be a differential graded algebra. For any $\eta, \xi \in$ $H H^{*}(A, A)$ and $m \in H H^{*}\left(A, A^{\vee}\right)$,

$$
\begin{aligned}
\{\xi, \eta\} \cdot m=(-1)^{|\xi|} B^{\vee} & {[(\xi \cup \eta) \cdot m]-\xi \cdot B^{\vee}(\eta \cdot m) } \\
& +(-1)^{(|\eta|+1)(|\xi|+1)} \eta \cdot B^{\vee}(\xi \cdot m)+(-1)^{|\eta|}(\xi \cup \eta) \cdot B^{\vee}(m) .
\end{aligned}
$$


Proof. - The action of $H H^{*}(A, A)$ on $H H_{*}(A, A)$ comes from a (right) action of the $\mathcal{C}^{*}(A, A)$ on $\mathcal{C}_{*}(A, A)$ given by $[6,(18)$ p. 82]

$$
\begin{gathered}
\mathcal{C}_{*}(A, A) \otimes \mathcal{C}^{*}(A, A) \rightarrow \mathcal{C}_{*}(A, A) \\
\left(m\left[a_{1}|\ldots| a_{n}\right], f\right) \mapsto i_{f}\left(m\left[a_{1}|\ldots| a_{n}\right]\right):=\sum_{p=0}^{n} \pm\left(m . f\left[a_{1}|\ldots| a_{p}\right]\right)\left[a_{p+1}|\ldots| a_{n}\right] .
\end{gathered}
$$

Here \pm is the Koszul sign $(-1)^{|f|\left(\left|a_{1}\right|+\ldots\left|a_{n}\right|+n\right)}$. Therefore $\mathcal{C}^{*}(A, A)$ acts on the left on the dual $\mathcal{C}_{*}(A, A)^{\vee}$. Explicitly, the action is given by

$$
\begin{gathered}
\mathcal{C}^{*}(A, A) \otimes \mathcal{C}_{*}(A, A)^{\vee} \rightarrow \mathcal{C}_{*}(A, A)^{\vee} \\
(f, \varphi) \mapsto(f . \varphi)
\end{gathered}
$$

defined by

$$
(f . \varphi)\left(m\left[a_{1}|\ldots| a_{n}\right]\right)=(-1)^{|f||\varphi|+|f||m|} \sum_{p=0}^{n} \varphi\left(\left(m . f\left[a_{1}|\ldots| a_{p}\right]\right)\left[a_{p+1}|\ldots| a_{n}\right]\right) .
$$

Through the canonical isomorphism $\mathcal{C}^{*}\left(A, A^{\vee}\right) \stackrel{\cong}{\rightrightarrows} \mathcal{C}_{*}(A, A)^{\vee}, g \mapsto \varphi$ defined by

$$
\varphi\left(m\left[a_{1}|\ldots| a_{n}\right]\right):=(-1)^{|m|\left(\left|a_{1}\right|+\ldots\left|a_{n}\right|+n\right)}\left(g\left[a_{1}|\ldots| a_{n}\right]\right)(m),
$$

this left action coincides with the left action defined by the composite (10).

Let us be precise about our sign convention: we define $B^{\vee}$ by $B^{\vee}(m):=$ $(-1)^{|m|} m \circ B$. Denote by $\varepsilon$ the $\operatorname{sign}(-1)^{|m|(|\xi|+|\eta|+1)}$. For any $m \in$ $H H_{*}(A, A)^{\vee}$, we have the following equalities:

$$
\begin{gathered}
m(\{\xi, \eta\} \cdot c)=\varepsilon(\{\xi, \eta\} \cdot m)(c), \\
(-1)^{|\xi|} m \circ B[(\xi \cup \eta) \cdot c]=(-1)^{|\xi|+|m|}\left[B^{\vee}(m)\right][(\xi \cup \eta) \cdot c]=\varepsilon(-1)^{|\eta|}\left[(\xi \cup \eta) \cdot B^{\vee}(m)\right](c), \\
-m[\xi \cdot B(\eta \cdot c)]=(-1)^{1+|m||\xi|}[\xi \cdot m] \circ B(\eta \cdot c)= \\
(-1)^{1+|m||\xi|+|\xi|+|m|}\left[B^{\vee}(\xi \cdot m)\right](\eta \cdot c)=\varepsilon(-1)^{(|\eta|+1)(|\xi|+1)}\left[\eta \cdot B^{\vee}(\xi \cdot m)\right](c),
\end{gathered}
$$

by exchanging $\xi$ and $\eta$,

$$
\begin{gathered}
(-1)^{(|\eta|+1)(|\xi|+1)} m[\eta \cdot B(\xi \cdot c)]=-\varepsilon\left[\xi \cdot B^{\vee}(\eta \cdot m)\right](c), \\
(-1)^{|\eta|} m[(\xi \cup \eta) \cdot B(c)]=\varepsilon(-1)^{|\eta|+|m|}[(\xi \cup \eta) \cdot m] \circ B(c)= \\
\varepsilon(-1)^{|\xi|} B^{\vee}[(\xi \cup \eta) \cdot m](c) .
\end{gathered}
$$

Therefore by evaluating the linear form $m \in H H_{*}(A, A)^{\vee}$ on the terms of the equation given by Lemma 15 , we obtain the desired equality. 
REMARK 17. - The equality in Lemma 16 is the same as the equality in Lemma 15. In fact, alternatively, to prove Lemma 16, we could have proved that the Gerstenhaber algebra $H H^{*}(A, A)$ and the dual of Connes boundary map $B^{\vee}$ on $H H^{*}\left(A, A^{\vee}\right)$ form a calculus. Indeed, in the proof of Lemma 15, we have remarked that the desired equality holds for any calculus.

Proof of Proposition 12. - By definition the $\Delta$ operator on $H H^{*}(A, A)$ is given by $(\Delta a) \cdot m:=B^{\vee}(a . m)$ for any $a \in H H^{*}(A, A)$. Therefore the proposition follows from Lemma 16.

\section{Applications}

As first application of Proposition 12, we show

TheOREM 18. - Let $A$ be an algebra equipped with a degree $d$ quasiisomorphism of $A$-bimodules $\Theta: A \stackrel{\simeq}{\rightrightarrows} A^{\vee}$ between $A$ and its dual $\operatorname{Hom}(A, \mathbb{F})$. Then the Connes coboundary map on $H^{*}\left(A, A^{\vee}\right)$ defines via the isomorphism $H H^{*}(A, \Theta): H H^{p}(A, A) \stackrel{\cong}{\rightrightarrows} H H^{p-d}\left(A, A^{\vee}\right)$ a structure of Batalin-Vilkovisky algebra on the Gerstenhaber algebra $H^{*}(A, A)$.

In representation theory [7], an (ungraded) algebra $A$ is symmetric if $A$ is equipped with an isomorphism of $A$-bimodules $\Theta: A \cong A^{\vee}$ between $A$ and its dual $\operatorname{Hom}(A, \mathbb{F})$. The following Corollary is implicit in [26] and was for the first time explicited in [23, Theorem 1.6].

Corollary 19 ([26, 23]). - Let $A$ be a symmetric algebra. Then the Gerstenhaber algebra $H H^{*}(A, A)[16]$ is a Batalin-Vilkovisky algebra.

In [20] or [28, Corollary 3.4] or [5, Section 1.4] or [21, Theorem B] or [22, Section 11.6] or [18], this Batalin-Vilkovisky algebra structure on $H H^{*}(A, A)$ extends to a structure of algebra on the Hochschild cochain complex $\mathcal{C}^{*}(A, A)$ over various operads or PROPs: the so-called cyclic Deligne conjecture.

Proof of Theorem 18. - Let $\varepsilon_{A}: P:=B(A ; A ; A) \stackrel{\simeq}{\rightrightarrows} A$ be the bar resolution

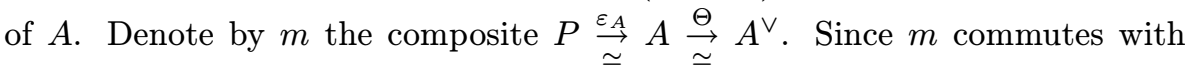
the differential, $m$ is a cycle in $\operatorname{Hom}_{A \otimes A^{\text {op }}}(P, A)$. As we saw in the proof of Proposition 11, the composite $H H^{*}(A, m) \circ H H^{*}\left(A, \varepsilon_{A}\right)^{-1}$ :

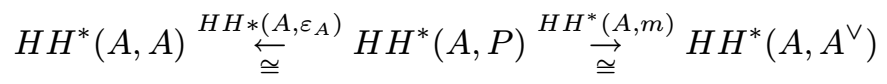

coincides with the morphism of left $H H^{*}(A, A)$-modules

$$
H H^{p}(A, A) \stackrel{\cong}{\rightarrow} H H^{p-d}\left(A, A^{\vee}\right), a \mapsto a \cdot m .
$$

By definition of $m$, this composite is also $H H^{*}(A, \Theta)$. 
Denote by by $\varepsilon_{B A}: T s A \rightarrow \mathbb{F}$ the canonical projection whose kernel is $T^{+} s A$. Since $\varepsilon_{A}: B(A ; A ; A) \rightarrow A$ is the composite of $A \otimes \varepsilon_{B A} \otimes A$ and of the multiplication on $A$

$$
A \otimes T s A \otimes A \rightarrow A \otimes \mathbb{F} \otimes A \cong A \otimes A \rightarrow A,
$$

the canonical isomorphisms of complexes

$$
\operatorname{Hom}_{A \otimes A^{\text {op }}}\left(B(A ; A ; A), A^{\vee}\right) \cong \mathcal{C}^{*}\left(A, A^{\vee}\right) \cong \mathcal{C}_{*}(A, A)^{\vee}
$$

map $m$ to the linear form on $\mathcal{C}_{*}(A, A)$ :

$$
\Theta(1) \otimes \varepsilon_{B A}: A \otimes T s A \rightarrow \mathbb{F} \otimes \mathbb{F} \cong \mathbb{F} .
$$

Connes (normalized or not) boundary map $B: \mathcal{C}_{*}(A, A) \rightarrow \mathcal{C}_{*}(A, A)$ factorizes through $A \otimes T^{+} s A$. So $B^{\vee}\left(\Theta(1) \otimes \varepsilon_{B A}\right)= \pm\left(\Theta(1) \otimes \varepsilon_{B A}\right) \circ B=0$. Therefore, we can apply Proposition 12 .

Remark: In the case of Corollary 19, $m$ corresponds to a trace $\Theta(1) \in$ $\mathcal{C}^{0}\left(A, A^{\vee}\right)$. Since $H\left(B^{\vee}\right): H H^{p}\left(A, A^{\vee}\right) \rightarrow H H^{p-1}\left(A, A^{\vee}\right)$ decreases (upper) degrees and $H H^{p}\left(A, A^{\vee}\right)=0$ for $p<0$, it is obvious that $H\left(B^{\vee}\right)(\Theta(1))=$ 0 .

Working, with rational coefficients, we easily obtain

Corollary 20 ([29]). — The Hochschild cohomology

$$
H H^{*}\left(S^{*}(M ; \mathbb{Q}), S^{*}(M ; \mathbb{Q})\right) \cong H H^{*-d}\left(S^{*}(M ; \mathbb{Q}), S^{*}(M ; \mathbb{Q})^{\vee}\right)
$$

is a Batalin-Vilkovisky algebra which extends the Gerstenhaber algebra from [16].

Tradler and Zeinalian [29] give a proof of this result. Here is a shorter proof, although we don't claim that we have obtained the same Batalin-Vilkovisky algebra.

Proof of Corollary 20. - Since we are working over $\mathbb{Q}$, there exists quasiisomorphisms of algebras [10, Corollary 10.10]

$$
S^{*}(M ; \mathbb{Q}) \stackrel{\simeq}{\rightrightarrows} D(M) \stackrel{\simeq}{\leftrightarrows} A_{P L}(M)
$$

where $A_{P L}(M)$ is a commutative (differential graded) algebra. Since the Gerstenhaber algebra structure on Hochschild cohomology is preserved by quasiisomorphism of algebras [11, Theorem 3], we obtain an isomorphism of Gerstenhaber algebras

$$
H H^{*}\left(S^{*}(M ; \mathbb{Q}), S^{*}(M ; \mathbb{Q})\right) \cong H H^{*}\left(A_{P L}(M), A_{P L}(M)\right) .
$$

Since $H\left(A_{P L}(M)\right) \cong H^{*}(M ; \mathbb{Q})$, Poincaré duality induces a quasi-isomorphism of $A_{P L}(M)$-modules

$$
A_{P L}(M) \stackrel{\simeq}{\rightarrow} A_{P L}(M)^{\vee}
$$


Since the algebra $A_{P L}(M)$ is commutative, this is in fact a quasi-isomorphism of $A_{P L}(M)$-bimodules. By applying Theorem 18, we obtain that

$$
H H^{*}\left(A_{P L}(M), A_{P L}(M)\right) \cong H H^{*-d}\left(A_{P L}(M), A_{P L}(M)^{\vee}\right)
$$

is a Batalin-Vilkovisky algebra.

In [12] and [3, Theorem 5.4], it is shown that the Batalin-Vilkovisky algebra $H_{p+d}(L M ; \mathbb{Q})$ of Chas and Sullivan is isomorphic to the Batalin-Vilkovisky algebra on

$$
H H^{-p}\left(S^{*}(M ; \mathbb{Q}), S^{*}(M ; \mathbb{Q})\right) \cong H H^{-p-d}\left(A_{P L}(M), A_{P L}(M)^{\vee}\right) .
$$

given by Corollary 20. Note that this Batalin-Vilkovisky algebra on $H H^{*}\left(A_{P L}(M), A_{P L}(M)\right)$ depends only of the commutative algebra $A_{P L}(M)$ and of the fundamental class $[M] \in H_{d}\left(A_{P L}(M)^{\vee}\right) \cong H_{d}(M)$. So this BatalinVilkovisky algebra can be computed using Sullivan models. On the contrary, the Batalin-Vilkovisky algebra given by our main theorem (Theorem 22) seems hard to compute. As we would like to emphasize in this paper, the rational case is much more simple than the case of a field $\mathbb{F}$ of characteristic $p$ different from 0. Nevertheless, we expect that the Batalin-Vilkovisky algebra given by Corollary 20 coincides with the Batalin-Vilkovisky algebra given by our main theorem (Theorem 22) in the case of the field $\mathbb{Q}$.

Recall the following theorem due to Félix, Thomas and Vigué-Poirrier.

ThEOREM 21 ([13, Theorem 13]). - Let $M$ be a compact connected oriented d-dimensional smooth manifold. Then there is an isomorphism of lower degree d

$$
\mathbb{D}^{-1}: H H^{p}\left(S^{*}(M), S^{*}(M)\right) \stackrel{\cong}{\rightrightarrows} H H^{p-d}\left(S^{*}(M), S^{*}(M)^{\vee}\right) .
$$

As second application of Propositions 11 and 12, we will recover the isomorphism of Félix, Thomas and Vigué-Poirrier and prove our main theorem:

THEOREM 22. - Let $M$ be a compact connected oriented d-dimensional smooth manifold. Let $[M] \in H_{d}(M)$ be its fundamental class. Then

1) For any $a \in H H^{*}\left(S^{*}(M), S^{*}(M)\right)$, the image of a by $\mathbb{D}^{-1}$ is given by the action of a on $\left(J \circ H_{*}(s)\right)([M])$ :

$$
\mathbb{D}^{-1}(a)=a \cdot\left(J \circ H_{*}(s)\right)([M]) .
$$

2) The Gerstenhaber algebra structure on $H H^{*}\left(S^{*}(M), S^{*}(M)\right)$ and Connes coboundary map $H\left(B^{\vee}\right)$ ) on $H H^{*}\left(S^{*}(M), S^{*}(M)^{\vee}\right)$ defines via the isomorphism $\mathbb{D}^{-1}$ a structure of Batalin-Vilkovisky algebra. 
Here $s$ denotes $s: M \hookrightarrow L M$ the inclusion of the constant loops into $L M$. Recall that $J: H_{*}(L M) \rightarrow H H^{*}\left(S^{*}(M), S^{*}(M)^{\vee}\right)$ is the morphism introduced by Jones in [19]. If $M$ is supposed to be simply connected, then $J$ is an isomorphism.

Proof of Theorem 21 and of Theorem 22. - We first follow basically [13, Section 7]. Denote by $e v: L M \rightarrow M, l \mapsto l(0)$ the evaluation map. The morphism $J$ of Jones fits into the commutative triangle.

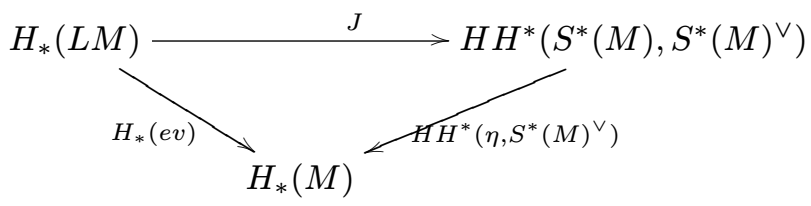

Since $s$ is a section of the evaluation map ev, $J \circ H_{*}(s)$ is a section of $H H^{*}\left(\eta, S^{*}(M)^{\vee}\right)$. Therefore $H H^{*}\left(\eta, S^{*}(M)^{\vee}\right) \circ J \circ H_{*}(s)([M])=[M]$.

By Poincaré duality, the composite of the two morphisms of $H^{*}(M)$-module

$$
H^{*}(M) \stackrel{\cap[M]}{\rightarrow} H_{*}(M) \cong H\left(S^{*}(M)^{\vee}\right), a \mapsto a \cap[M] \mapsto a \cdot[M] .
$$

is an isomorphism of lower degree $d$. Therefore by applying Proposition 11 to $[m]:=J \circ H_{*}(s)([M])$, we obtain Theorem 21 and part 1) of Theorem 22.

Consider $M$ equipped with the trivial $S^{1}$-action. The section $s: M \hookrightarrow$ $L M$ is $S^{1}$-equivariant. Therefore $\Delta\left(H_{*}(s)([M])\right)=0$. Recall that the Jones morphism $J$ satisfies $J \circ \Delta=H_{*}\left(B^{\vee}\right) \circ J$. Therefore, since $\left(H_{*}\left(B^{\vee}\right) \circ J \circ\right.$ $\left.H_{*}(s)\right)([M])=0$, by applying Proposition 12, we obtain part 2) of Theorem 22.

REMARK 23. - Part 1) of Theorem 22 means exactly that the morphism

$$
\mathbb{D}^{-1}: H H^{p}\left(S^{*}(M), S^{*}(M)\right) \stackrel{\cong}{\rightrightarrows} H H^{p-d}\left(S^{*}(M), S^{*}(M)^{\vee}\right)
$$

is the unique morphism of $H H^{*}\left(S^{*}(M), S^{*}(M)\right)$-modules such that the composite

$$
J^{-1} \circ \mathbb{D}^{-1}: H H^{-p}\left(S^{*}(M), S^{*}(M)\right) \stackrel{\cong}{\rightrightarrows} H_{p+d}(L M)
$$

respects the units of the algebras. We conjecture (Conjecture 4) that $J^{-1} \circ \mathbb{D}^{-1}$ respects also the products.

In [2] together with David Chataur, we give a third application of Propositions 11 and 12: 
Theorem 24 ([2, Theorem 24]). - Let $G$ be a connected compact Lie group of dimension d. Denote by $S_{*}(G)$ the algebra of singular chains of $G$. Consider Connes coboundary map $H\left(B^{\vee}\right)$ on the Hochschild cohomology of $S_{*}(G)$ with coefficients in its dual, $H H^{*}\left(S_{*}(G) ; S^{*}(G)\right)$. Then there is an isomorphism of graded vector spaces of upper degree $d$

$$
\mathbb{D}^{-1}: H H^{p}\left(S_{*}(G) ; S_{*}(G)\right) \stackrel{\cong}{\rightarrow} H H^{p+d}\left(S_{*}(G) ; S^{*}(G)\right)
$$

such that the Gerstenhaber algebra $H^{*}\left(S_{*}(G) ; S_{*}(G)\right)$ equipped with the operator $\Delta=\mathbb{D} \circ H\left(B^{\vee}\right) \circ \mathbb{D}^{-1}$ is a Batalin-Vilkovisky algebra.

\section{Cyclic homology}

In this section, we prove

THEOREM 25. - Let $M$ be a compact oriented smooth d-dimensional manifold. Then the negative cyclic cohomology on the singular cochains of $M$, $H C_{-}^{*}\left(S^{*}(M)\right)$, is a graded Lie algebra of lower degree $2-d$.

If $M$ is simply-connected, Jones [19] proved that there is an isomorphism

$$
H_{*}^{S^{1}}(L M) \stackrel{\cong}{\rightrightarrows} H C_{-}^{*}\left(S^{*}(M)\right) \text {. }
$$

In [1], Chas and Sullivan defined a Lie bracket, called the string bracket

$$
\{,\}: H_{p}^{S^{1}}(L M) \otimes H_{q}^{S^{1}}(L M) \rightarrow H_{p+q+2-d}^{S^{1}}(L M)
$$

Of course, we expect the two a priori different brackets to be related:

Conjecture 26. - The Jones isomorphism

$$
H_{*}^{S^{1}}(L M) \stackrel{\cong}{\rightrightarrows} H C_{-}^{*}\left(S^{*}(M)\right)
$$

is an isomorphism of graded Lie algebras between Chas-Sullivan string bracket and the Lie bracket defined in Theorem 25.

Theorem 25 follows directly from Theorem 22 and from the following proposition. In [23, Corollary 1.7 and Section 7], we proved that if $A$ is a symmetric algebra then its negative cyclic cohomology $H C_{-}^{*}(A)$ is a graded Lie algebra of lower degree 2 . In fact, we proved more generally

Proposition 27. - If the Hochschild cohomology of a (differential graded) algebra $A, H H^{*}\left(A, A^{\vee}\right)$, equipped with $H_{*}\left(B^{\vee}\right)$, has a Batalin-Vilkovisky algebra structure of degree $-d$ then its negative cyclic cohomology $\mathrm{HC}_{-}^{*}(A)$ is a graded Lie algebra of lower degree $2-d$. 
Proof. - Apply [23, Proposition 7.1] to the mixed complex $\mathcal{C}^{*}\left(A, A^{\vee}\right)$ (desuspended $d$-times in order to take into account the degree $d$ shift). By definition, $H C_{-}^{*}(A)$ is the differential torsion product

$$
\operatorname{Tor}^{H_{*}\left(S^{1}\right)}\left(\mathcal{C}^{*}\left(A, A^{\vee}\right), \mathbb{F}\right) \text {. }
$$

Another interesting particular case of [23, Proposition 7.1] is the following proposition.

Proposition 28. - If the Hochschild homology of an algebra $A, H H_{*}(A, A)$, equipped with Connes boundary map B, has a Batalin-Vilkovisky algebra structure then its cyclic homology $\mathrm{HC}_{*}(A)$ is a graded Lie algebra of lower degree 2.

Proof. - Apply [23, Proposition 7.1] to the mixed complex $\mathcal{C}_{*}(A, A)$. By definition, $H C_{*}(A)$ is the differential torsion product

$$
\operatorname{Tor}^{H_{*}\left(S^{1}\right)}\left(\mathcal{C}_{*}(A, A), \mathbb{F}\right) \text {. }
$$

Remark that in fact, these graded Lie algebra structures extend to Lie $_{\infty^{-}}$ algebra structures like the Chas-Sullivan string bracket [1, Theorem 6.2 and Corollary 6.3].

Chas-Sullivan string bracket is defined using Gysin long exact sequence. The bracket given by Theorem 25 is defined similarly using Connes long exact sequence. Jones [19] proved that Gysin and Connes long exact sequences are isomorphic. Therefore Conjecture 4 implies Conjecture 26, since as we explained in the introduction, Conjecture 4 implies that the Jones isomorphism

$$
J: H_{p+d}(L M) \stackrel{\cong}{\rightrightarrows} H H^{-p-d}\left(S^{*}(M), S^{*}(M)^{\vee}\right)
$$

is an isomorphism of Batalin-Vilkovisky algebras.

\section{BIBLIOGRAPHY}

[1] M. Chas \& D. Sullivan - "String topology", preprint arXiv:math/9911159, 1999.

[2] D. Chataur \& L. Menichi - "String topology of classifying spaces", preprint arXiv:math.AT/0801.0174, 2008.

[3] X. Chen - "On a general chain model of the free loop space and string topology", preprint arXiv:0708.1197, 2007.

[4] R. L. Cohen \& J. D. S. Jones - "A homotopic theoretic realization of string topology", Math. Ann. 324 (2002), p. 773-798.

[5] K. Costello - "Topological conformal field theories and Calabi-Yau categories", Adv. Math. 210 (2007), p. 165-214. 
[6] J. Cuntz, G. Skandalis \& B. Tsygan - Cyclic homology in noncommutative geometry, Encyclopaedia of Mathematical Sciences, vol. 121, Springer, 2004.

[7] C. Curtis \& I. Reiner - Methods of representation theory, vol. 1, J. Wiley and Sons, New York, 1981.

[8] C.-H. Eu \& T. SchedleR - "Calabi-Yau Frobenius algebras", preprint arXiv:0710.3391, 2007.

[9] Y. FÉlix, S. Halperin \& J.-C. Thomas - "Gorenstein spaces", Adv. in Math. 71 (1988), p. 92-112.

[10] _ Rational homotopy theory, Graduate Texts in Math., vol. 205, Springer, 2000.

[11] Y. Félix, L. Menichi \& J.-C. Thomas - "Gerstenhaber duality in Hochschild cohomology", J. Pure Appl. Algebra 199 (2005), p. 43-59.

[12] Y. FÉlix \& J.-C. Thomas - "Rational BV-algebra in string topology", preprint arXiv:0705.4194, 2007.

[13] Y. Félix, J.-C. Thomas \& M. Vigué-Poirrier - "The Hochschild cohomology of a closed manifold", Publ. Math. Inst. Hautes Études Sci. 99 (2004), p. 235-252.

[14] _ "Rational string topology", J. Eur. Math. Soc. (JEMS) 9 (2007), p. $123-156$.

[15] I. M. Gel'FAnd, Y. L. DAletskil̆ \& B. Tsygan - "On a variant of noncommutative differential geometry", Dokl. Akad. Nauk SSSR 308 (1989), p. $1293-1297$.

[16] M. Gerstenhaber - "The cohomology structure of an associative ring", Ann. of Math. 78 (1963), p. 267-288.

[17] V. GinzBurg - "Calabi-yau algebras", preprint arXiv:math/0612139v3, 2006.

[18] P. Hu - "The Hochschild cohomology of a Poincaré algebra", preprint arXiv:0707.4118, 2007.

[19] J. D. S. Jones - "Cyclic homology and equivariant homology", Invent. Math. 87 (1987), p. 403-423.

[20] R. M. KaUfmann - "A proof of a cyclic version of Deligne's conjecture via Cacti", preprint arXiv:math.QA/0403340, 2004.

[21] _ "Moduli space actions on the Hochschild co-chains of a Frobenius algebra. I. Cell operads", J. Noncommut. Geom. 1 (2007), p. 333-384.

[22] M. Kontsevich \& Y. Soibelman - "Notes on A-infinity algebras, A-infinity categories and non-commutative geometry. I", preprint arXiv:math.RA/0606241, 2006.

[23] L. MENiCHI - "Batalin-Vilkovisky algebras and cyclic cohomology of Hopf algebras", K-Theory 32 (2004), p. 231-251. 
[24] _ "String topology for spheres", to appear in Comment. Math. Helv.. [25] S. Merkulov - "De Rham model for string topology", Int. Math. Res. Not. 55 (2004), p. 2955-2981.

[26] T. TRADLER - "The BV algebra on Hochschild cohomology induced by infinity inner products", preprint arXiv:math.QA/0210150, 2002.

[27] T. Tradler \& M. Zeinalian - "Algebraic string operations", preprint arXiv:math/0605770, 2006.

[28] _ "On the cyclic Deligne conjecture", J. Pure Appl. Algebra 204 (2006), p. 280-299.

[29] , "Infinity structure of Poincaré duality spaces", Algebr. Geom. Topol. 7 (2007), p. 233-260. 